\title{
Zehn Maximen für Veränderer
}

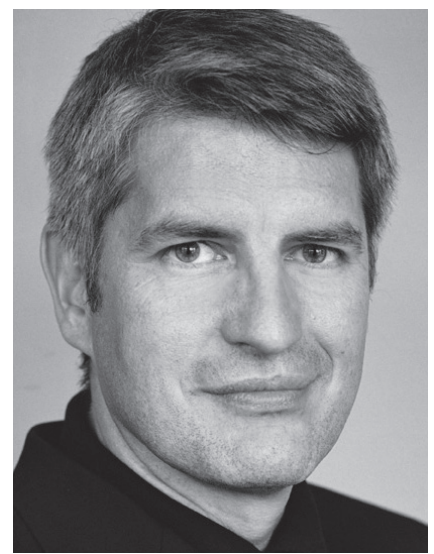

VON GEORG KRAUS

Dr. Georg Kraus ist geschäftsführender Gesellschafter der Unternehmensberatung Dr. Kraus \& Partner in Bruchsal, für die fast 50 Trainer, Berater und Coachs arbeiten. Er ist Autor des "Change Management Handbuches" (Cornelsen Verlag, 2004) sowie zahlreicher ProjektmanagementBücher.

Internet http://www.kraus-undpartner.de

\author{
Soziale Organisationen und Unternehmen der \\ Sozialwirtschaft müssen sich immer wieder erneuern, \\ wenn sie ihren Aufgaben konzeptionell, fachlich \\ und wirtschaftlich angemessen gerecht werden \\ wollen. Doch in vielen solcher Prozesse lauern \\ Fallstricke, die Führungskräfte kennen sollten.
}

Veränderungsprozesse planen und steuern - dies ist heute eine Herausforderung, vor der Manager fast täglich stehen. Deshalb müssen sie auch in Sachen Change-Management fit sein. Einige Tipps mögen helfen, worauf Sie dabei achten sollten.

1 Nicht jede Veränderung ist ein - Change-Prozess. Der Begriff »Change« ist ein Modebegriff. Entsprechend inflationär wird er gebraucht. Ganz gleich, ob Unternehmen ihre Büros neu streichen oder ob Verbände fusionieren, fast jede Veränderung wird heute als »Change" tituliert. Dies verursacht vielfach Verwirrung.

Tipp: Bezeichnen Sie als »ChangeProzess« nur Entwicklungen, die auch einen kulturellen Wandel in Ihrer Organisation erfordern - also bei denen Ihre Mitarbeitenden (und Sie!) Gewohntes über Bord werfen und neue Denk- und Verhaltensweisen entwickeln müssen.

7 Rom wurde nicht in einer Nacht - erbaut. Den meisten Menschen fällt es schwer, gewohnte Denk- und Verhaltensmuster aufzugeben. Denn diese vermitteln ihnen auch Sicherheit. Entsprechend langwierig sind zumeist Prozesse, bei denen ganze Mitarbeitergruppen ihr Verhalten verändern müssen. Von heute auf morgen geht das nicht.

Tipp: Bedenken Sie die erforderlichen Zeiträume beim Planen von Change-
Projekten. Sonst definieren Sie unrealistische Ziele.

3 Struktur und Kultur bedingen sich 3. wechselseitig. Auch wenn nicht jede Veränderung ein Change-Prozess ist, so finden in Unternehmen doch mehr Change-Prozesse statt, als insbesondere deren »Techniker« häufig vermuten. Sie denken oft "Wir führen doch nur ein neues System des Qualitätsmanagements ein « und übersehen, dass sich hierdurch die Arbeitsinhalte und Arbeitsbeziehungen der Mitarbeiterinnen und Mitarbeiter oft tiefgreifend verändern. Entsprechend überrascht sind sie, wenn die Kolleginnen und Kollegen plötzlich mit (vielleicht verdecktem) Widerstand reagieren.

Tipp: Analysieren Sie, wenn größere Veränderungen anstehen, deren Auswirkungen für die Mitarbeitenden, damit nicht unverhofft ein Orkan über Sie hinwegfegt, der das gesamte Projekt lahmlegt.

4. Was beschlossen ist, ist noch - lange nicht umgesetzt. Viele Unternehmensführer agieren bei ChangeProjekten wie folgt: Sie fassen die wegweisenden Beschlüsse. Dann rufen sie eine Projektgruppe ins Leben, die ihre Beschlüsse realisieren soll, und anschließend wenden Sie sich neuen Aufgaben zu. Wenn Sie so vorgehen, ist Ihr Projekt von vornherein gescheitert. Warum? Mitarbeiterinnen und Mitarbeiter orien- 
tieren ihr Verhalten an dem der oberen Führungskräfte. Nur wenn von ihnen immer wieder das Signal ausgeht »Die Veränderung ist nötig und an ihr führt kein Weg vorbei«, lässt sich in Unternehmen die nötige Veränderungsenergie erzeugen.

Tipp: Zeigen Sie Präsenz. Werben Sie immer wieder für die Veränderung - selbst wenn Sie die Verantwortung für das Umsetzen einer Projektgruppe übertragen haben.

5. Bei jeder Veränderung gibt es Verlierer. Unternehmen neigen dazu, alles in ein rosarotes Licht $\mathrm{zu}$ tauchen - auch Veränderungsvorhaben. Sie werden den Mitarbeitenden meist so präsentiert, als ob es in ihnen nur Gewinner gäbe. Doch Mitarbeiterinnen und Mitarbeiter sind nicht dumm. Sie wissen: Nur Gewinner gibt es so gut wie nie. Zumindest gibt es bei jedem ChangeProjekt auch Mitarbeitende, die sich als Verlierer empfinden, beispielsweise weil sie Einfluss oder Privilegien verlieren. Oder weil sie neue Aufgaben wahrnehmen müssen.

Tipp: Sprechen Sie offen und ehrlich mit Ihren Mitarbeiterinnen und Mitarbeitern über mögliche Folgen und geben Sie ihnen Raum, ihre Bedenken zu artikulieren. Sonst verdichten sich diese zu massiven Widerständen.

6. »Lonely heroes « stehen auf verlorenem Posten. Auch Ihre Einrichtungsleiter und andere Führungskräfte sind nur Menschen - und letztlich normale Arbeitnehmer. Deshalb sollten Sie es nicht als selbstverständlich erachten, dass alle Führungskräfte die von Ihnen gewünschten Veränderungen von vornherein mittragen. Zugleich sind Sie aber auf deren Unterstützung angewiesen, wenn es darum geht, die Veränderungen in deren Bereichen umzusetzen.

Tipp: Versuchen Sie, bevor Sie ein Change-Projekt verkünden, möglichste viele Führungskräfte als Mitstreiter zu gewinnen, zum Beispiel, indem sie diese in Ihre Entscheidung einbinden oder indem Sie diese in persönlichen Gesprächen ausführlich über die Gründe für Ihre Entscheidung und deren voraussichtliche Konsequenzen informieren.

7. Projektmanager brauchen Rückgrat und Erfahrung. Unternehmen übertragen die Verantwortung für Change-
Projekte oft jungen Führungskräften, als Chance sich zu gewähren - getreu dem Motto »Lass das mal den Mayer machen. Dann kann er zeigen, was in ihm steckt.« Als Folge hiervon werden die Projekte oft von Personen gemanagt, welche die Auswirkungen gewisser Entscheidungen und Handlungen auf die Organisation nur bedingt einschätzen können; von Personen zudem, die noch ein schwaches Standing in der Organisation haben. Entsprechend schwer fällt es ihnen, von den »Bereichsfürsten" die nötige Unterstützung zu erlangen - vor allem, wenn diese den ehrgeizigen Nachwuchs als Konkurrenz erfahren.

Tipp: Übertragen Sie die Verantwortung für strategische (Change-) Projekte gestandenen Führungskräften oder erfahrenen Projektmanagern. Oder stellen sie dem "Youngster « zumindest eine entsprechende Person als Coach zu Seite, mit der er nicht nur seine strategische, sondern auch taktische Marschroute austüfteln kann.

8. Nach dem Aufbruch folgt der lan- ge Marsch durch die Wüste. Oft starten Unternehmen voller Euphorie ein Projekt. Doch nach einiger Zeit beginnt das Jammern und Hadern. "Das bringt alles nichts.« $\gg \mathrm{Da}$ ändert sich ja sowieso nichts." Das ist normal - weil sich kulturelle Veränderungen nur in kleinen Schritten vollziehen.

Tipp: Rechnen Sie damit, dass es Probleme beim Umsetzen gibt. Werben Sie gerade beim anstrengenden »Marsch durch die Wüste« stark für die Veränderung, sonst erlahmt die Veränderungsenergie und die Mitarbeitenden fallen in ihre alten Verhaltensmuster zurück.

9. Der Weg zum Misserfolg ist mit - guten Vorsätzen gepflastert. Oft erlahmt die (Veränderungs-) Energie auch, weil die Mitarbeitenden beim Ausprobieren der neuen Verhaltenmuster registrieren »So, wie wir das früher gemacht haben, ging alles schneller und einfacher.« Warum? Sie haben noch keine neuen (Denk- und) Verhaltensroutinen entwickelt. Hinzu kommt: Bei jedem größeren Veränderungsprojekt ist in einer Übergangszeit zunächst einmal Sand im Getriebe der Organisation.

Tipp: Machen Sie Ihren Führungskräften bewusst, wie wichtig es gerade in dieser Übergangsphase ist, dass sie ihre Mitarbeiterinnen und Mitarbeiter führen - und stellen Sie den Mitarbeitenden Unterstützer zur Seite stellen, die diese immer wieder neu motivieren.

10. Zum Feiern gibt es fast immer einen Grund. Gerade weil der Weg zum großen Ziel bei Change-Projekten oft so weit ist, dass die Beteiligten zuweilen das Gefühl haben »Wir kommen nie ans Ziel«, ist es wichtig, dass Sie Etappenziele formulieren, deren Erreichen gefeiert werden kann. Das macht den Beteiligten Mut.

Tipp: Ziehen Sie, wenn es was zu feiern gibt, als »Chef « auch mal ganz spontan (oder geplant) die »Spendierhosen " an - und organisieren Sie zum Beispiel ein kleines Fest oder einen Ausflug. Denn nichts motiviert Mitarbeiter mehr, als wenn sie sehen, dass ihre Leistung gewürdigt wird. 